\title{
ACÚMULO DE MACRONUTRIENTES EM CANA-DE-AÇÚCAR EM FUNÇÃO DA ADUBAÇÃO NITROGENADA E DOS RESÍDUOS CULTURAIS INCORPORADOS AO SOLO NO PLANTIO ( $\left.{ }^{1}\right)$
}

\author{
HENRIQUE COUTINHO JUNQUEIRA FRANCO $\left(2^{*}\right)$; ISABELA RODRIGUES BOLOGNA $\left(^{3}\right)$; \\ CARLOS EDUARDO FARONI $\left({ }^{2}\right)$; ANDRÉ CESAR VITTI $\left({ }^{3}\right)$; PAULO CESAR OCHEUZE TRIVELIN $\left({ }^{3},{ }^{4}\right)$
}

\section{RESUMO}

Com o objetivo de avaliar o acúmulo de macronutrientes (N, P, K, Ca, Mg e S) na parte aérea e na parte subterrânea da cana-de-açúcar (cana-planta) em função da adubação nitrogenada e dos resíduos culturais incorporados ao solo, foi realizado um experimento em vasos contendo $250 \mathrm{~kg}$ de terra de textura arenosa. O delineamento experimental foi um fatorial $(4 \times 2)$ em blocos casualizados com três repetições. Os tratamentos corresponderam aos fatores: (1) Doses de N de 0, 900, 1800 e $2700 \mathrm{mg}^{\text {vaso }}{ }^{-1}$; (2) Restos culturais da última soca incorporados ao solo simulando a reforma do canavial, contendo folhas secas (CF) ou não (SF). O experimento foi realizado em Piracicaba (SP), de janeiro a dezembro de 1996. A adubação nitrogenada promoveu maior acúmulo de $\mathrm{N}$ e $\mathrm{S}$ na parte aérea das plantas de cana-de-açúcar, sendo a relação N/S igual a 2,0, proporcionando maiores acúmulos de todos os macronutrientes na parte subterrânea (raízes e rizomas) da cana-planta. Esse acúmulo seguiu a seguinte ordem decrescente de grandeza: $\mathrm{N}=\mathrm{K}>\mathrm{Ca}>\mathrm{Mg}=\mathrm{S}>\mathrm{P}$. Os tipos de resíduos culturais incorporados ao solo não proporcionaram efeito significativo no acúmulo de macronutrientes pela cultura de cana-de-açúcar durante a safra de cana-planta.

Palavras-chave: nitrogênio, raízes, Saccharum spp., sinergismo.

\author{
ABSTRACT \\ MACRONUTRIENTS ACCUMULATION IN SUGARCANE CROP RELATED TO NITROGEN \\ FERTILIZATION AND CULTURAL RESIDUES INCORPORATED TO THE SOIL AT PLANTING
}

With the aim to evaluate the accumulation of macronutrients $(\mathrm{N}, \mathrm{P}, \mathrm{K}, \mathrm{Ca}, \mathrm{Mg}$ and $\mathrm{S})$ in aboveground and below-ground sugarcane portions as a function of nitrogen fertilization and crop residues incorporated in the soil an experiment was developed in pots containing $250 \mathrm{~kg}$ of sandy soil. The experimental design was a randomized $(4 \times 2)$ blocks with three replications. The treatments were: $(1)$ Nitrogen rates of $0,900,1800$ and $2700 \mathrm{mg} \mathrm{pot}^{-1}$; (2) Crop residues of the last sugarcane ratoon crop incorporated to the soil, with dry leaves (CF) or without (SF). The study was carried out in Piracicaba,

$\left({ }^{1}\right)$ Recebido para publicação em 9 de setembro de 2005 e aceito em 14 de maio de 2007.

$\left({ }^{2}\right)$ Doutorando do Programa em Solos e Nutrição de Plantas da Escola Superior de Agricultura "Luiz de Queiroz" - ESALQ USP, Caixa Postal 9, 13418-900 Piracicaba (SP). Bolsista FAPESP. E-mail: hjfranco@cena.usp.br (*) Autor correspondente; cfaroni@cena.usp.br

$\left({ }^{3}\right)$ Laboratório de Isótopos Estáveis, Centro de Energia Nuclear na Agricultura, Universidade de São Paulo - CENA/USP, Caixa Postal 96, 13400-970 Piracicaba (SP). E-mail: acvitti@cena.usp.br; pcotrive@cena.usp.br

$\left({ }^{4}\right)$ Bolsista do CNPq. 
SP, from January to December 1996. Nitrogen fertilization promoted higher accumulation of $\mathrm{N}$ and $\mathrm{S}$ in the above-ground portion of sugarcane and the value of nitrogen sulphur ratio was 2. Nitrogen fertilization provided higher accumulation of all macronutrients in the below ground portion (root system) of sugarcane. This accumulation followed the decreasing order: $\mathrm{N}=\mathrm{K}>\mathrm{Ca}>\mathrm{Mg}=\mathrm{S}>\mathrm{P}$. The kinds of crop residues incorporated in the soil have not promoted significant effect on macronutrients accumulation by sugarcane.

Key words: nitrogen, roots, Saccharum spp., synergism.

\section{Introdução}

Desconhece-se o motivo pelo qual a cana-deaçúcar (cana-planta) é pouco responsiva à adubação nitrogenada. Entretanto, com as rebrotas (cana-soca) observa-se resposta na produção de colmos a essa prática (CARNAÚBA, 1990).

O tipo de resposta da cana-planta à adubação nitrogenada tem sido atribuído à fixação biológica do $\mathrm{N}$ atmosférico; às perdas por lixiviação de Nfertilizante; ao vigor do sistema radicular da cana-planta comparada ao de soqueiras; às condições climáticas, como temperatura e pluviosidade; à melhoria da fertilidade do solo, após a reforma dos canaviais, associada à calagem, ao preparo mecânico e à incorporação de restos da cultura anterior (Azeredo et al., 1986; Carnaúba, 1990; Orlando Filho et al., 1999; Urquiaga et al., 1992). Há de se salientar, ainda, que na reforma dos canaviais quantidades significativas $\left(10\right.$ a $\left.20 \mathrm{t} \mathrm{ha}^{-1}\right)$ de restos culturais (ponteiros, folhas secas, sistema radicular e rebrota de cana-de-açúcar), com relação C:N superior a 100 e conteúdo de $\mathrm{N}$ entre 40 e $80 \mathrm{~kg} \mathrm{ha}^{-1}$ (TRIVELIN et al., 1995; 1996), são incorporados ao sistema solo-planta. No entanto, quando a cana é queimada, os ponteiros e as palhas que permanecem no campo representam cerca de $30 \%$ a $50 \%$ da quantidade obtida de resíduos do canavial colhido sem queima (BASANTA et al., 2002). A maior parte do $\mathrm{N}$ contido nas folhas secas é perdida por volatilização ou na forma de aerosóis durante a queima do canavial. Portanto, o manejo sem despalha a fogo, potencialmente, conserva nitrogênio no sistema. Entretanto, os resultados de mineralização do $\mathrm{N}$ desses resíduos revelam que a liberação é lenta, variando entre $3 \%$ a $30 \%$ durante o ciclo seguinte da cultura (BASANTA et al., 2002; OLIVEIRA et al., 2002).

O aumento na produtividade da cana-deaçúcar (cana-planta) em resposta à adubação nitrogenada foi constatado por Silveira e CROCOMO (1990) em estudo de assimilação de $\mathrm{N}$ em cana-planta, na presença de elevada dose de N; por KoRNDÖRFER et al. (1997), com quatro cultivares de cana-de-açúcar em um Latossolo Vermelho-Amarelo álico, distrófico, de textura média; e por OrLANDo Filho et al. (1999), em um solo argiloso eutrófico.
Além de aumentar a produtividade de colmos de cana-de-açúcar, a adubação nitrogenada de plantio pode ainda gerar efeito sinérgico na absorção de outros nutrientes. Por outro lado, durante a senescência das folhas as proteínas dos cloroplastos são degradadas, e frações de nitrogênio podem ser deslocadas via floema para outros órgãos das plantas, tais como raízes e rizomas (Feller e FIsCher, 1994). Esse mecanismo poderá contar também com a contribuição de outros elementos, entre eles os macronutrientes móveis no floema $(\mathrm{P}, \mathrm{K} \mathrm{e} \mathrm{Mg})$.

A hipótese de direcionamento das reservas da planta para a parte subterrânea (raízes e rizomas) e posterior utilização pela cultura foi evidenciada por Trivelin et al. (1984). Esses autores verificaram que $7 \%$ do nitrogênio aplicado na parte aérea da planta foi translocado ao sistema radicular e, posteriormente, utilizado pela primeira rebrota da cana-de-açúcar. Possivelmente, essa translocação de nutrientes seja um mecanismo que garante o melhor vigor do sistema radicular e da rebrota para o próximo ciclo (TRIVELIN et al., 2002), motivo pelo qual a cana-de-açúcar deva ser considerada cultura perene e a denominação semiperene seja caracterizada apenas pela renovação periódica dos canaviais.

Neste contexto, a pesquisa teve como objetivo avaliar o acúmulo de macronutrientes na parte aérea e na subterrânea da cultura de cana-de-açúcar (canaplanta) em função da adubação nitrogenada e dos resíduos culturais incorporados no plantio.

\section{Material e Métodos}

O trabalho foi realizado em área experimental do Posto Meteorológico do Departamento de Ciências Exatas da ESALQ/USP, em Piracicaba, Estado de São Paulo. O delineamento experimental foi um fatorial $(4 \times 2)$ em blocos casualizados com três repetições. Os tratamentos corresponderam aos fatores: (1) Doses de $\mathrm{N}$ de $0,900,1800$ e $2700 \mathrm{mg} \mathrm{vaso}^{-1}$; (2) Restos culturais da última soca incorporados ao solo simulando a reforma do canavial, contendo folhas secas (CF) ou não (SF). O experimento constou de 24 parcelas constituídas por vasos de $0,60 \mathrm{~m}$ de diâmetro e 0,90 $\mathrm{m}$ de altura, contendo $250 \mathrm{~kg}$ de terra de textura arenosa $\left(840,60\right.$ e $100 \mathrm{~g} \mathrm{~kg}^{-1}$, respectivamente, para 
areia total, silte e argila) com as seguintes características químicas: 4,4 para $\mathrm{pH}\left(\mathrm{CaCl}_{2}\right) ; 19 \mathrm{~g} \mathrm{~kg}^{-}$ ${ }^{1}$ de MO; $10 \mathrm{mg} \mathrm{dm}^{-3}$ de $\mathrm{P}$ (resina), K, Ca, Mg e H+Al: $1,17,3$ e $34 \mathrm{mmol}_{\mathrm{c}} \mathrm{dm}^{-3}$ respectivamente.

Na camada superficial da terra dos vasos (0-0,25 m), aplicaram-se $\mathrm{CaO}$ e $\mathrm{MgO}$, na relação 2:1 em termos de massa, em quantidade suficiente para elevar a $60 \%$ a saturação por bases. Em seguida, incorporaram-se, nessa mesma profundidade, os resíduos culturais de cana-de-açúcar. No tratamento com incorporação de folhas secas (CF), observaramse colmos aéreos e ponteiros (122 g de matéria seca - MS - por vaso; 423 e $7,84 \mathrm{~g} \mathrm{~kg}^{-1}$ de C e N respectivamente); rizomas (251 g de MS por vaso; 400 e $5,70 \mathrm{~g} \mathrm{~kg}^{-1}$ de $\mathrm{C}$ e $\mathrm{N}$ respectivamente) e folhas secas (177 g de MS por vaso; 386 e 3,99 $\mathrm{g} \mathrm{kg}^{-1}$, de C e $\mathrm{N}$ respectivamente), quantidades equivalentes a 4,$3 ; 8,9$ e $6,3 \mathrm{t} \mathrm{ha}^{-1}$ de massa de matéria seca respectivamente, referidas com base em superfície, considerando-se a área dos vasos de $0,283 \mathrm{~m}^{2}$. Esses valores foram definidos tomando por base as produções médias de resíduos culturais observados por Trivelin et al. (1995). No tratamento com incorporação de resíduos culturais sem folhas secas (SF), os restos culturais (rizomas, colmos aéreos e ponteiros) foram misturados ao solo nas mesmas quantidades especificadas, e a quantidade equivalente de restos culturais foi de $13,2 \mathrm{t} \mathrm{ha}^{-1}$. Todos os restos culturais foram triturados antes de serem misturados ao solo dos vasos.

Dez dias após a adição do calcário e dos restos culturais, realizou-se a adubação de plantio. Em 8 de janeiro de 1996 transplantaram-se, para cada vaso, três mudas de cana-de-açúcar da cultivar SP80-1842, obtidas de nós de colmos tratados termicamente, com, aproximadamente, $0,20 \mathrm{~m}$ de altura. A adubação de plantio foi realizada no fundo de cova com $0,15 \mathrm{~m}$ de profundidade, aplicando-se 900; 1.800 e $2.700 \mathrm{mg}$ vaso $^{-1}$ de N-uréia. Inclui-se um tratamento testemunha sem aplicação de N. O cloreto de potássio e o superfosfato triplo também foram aplicados, nas doses equivalentes de $120 \mathrm{~kg} \mathrm{ha}^{-1}$ de $\mathrm{K}_{2} \mathrm{O}$ e $\mathrm{P}_{2} \mathrm{O}_{5}$ respectivamente.

Durante o período experimental, que se estendeu de 8 de janeiro a 9 de dezembro de 1996, o volume total de água recebido pela cultura, de precipitações pluviais e irrigações nas épocas de estiagem, foi de $2.014 \mathrm{~mm}$. A colheita da parte aérea e da parte subterrânea foi realizada 11 meses após o plantio. Após a secagem do material vegetal em estufa de circulação forçada de ar $\left(65^{\circ} \mathrm{C}\right)$, as amostras de cada parte da planta foram moídas e determinadas as concentrações de $\mathrm{N}, \mathrm{P}, \mathrm{K}, \mathrm{Ca}, \mathrm{Mg}$ e $\mathrm{S}$ conforme Malavolta et al. (1989). Os resultados foram submetidos à análise da variância em delineamento de blocos casualizados, utilizando-se o teste $\mathrm{F}$ a $5 \%$ de probabilidade. Para comparar o efeito de doses de $\mathrm{N}$ realizou-se o estudo de regressão (linear e quadrática).

\section{Resultados e Discussão}

Os resultados de produção de massa de material seco da parte aérea e subterrânea (sistema radicular) utilizados para o cálculo de acúmulo de macronutrientes pela cultura de cana-de-açúcar, no presente trabalho, foram publicados por TRIVELIN et al. (2002).

Não foi observado efeito dos tipos de resíduos culturais incorporados ao solo nos resultados de produção de matéria seca $\left(\mathrm{F}=1,7^{\mathrm{ns}} ; \mathrm{C} . \mathrm{V} .=8 \%\right)$, havendo efeito das doses de $\mathrm{N}$, com resposta linear na parte subterrânea $\left(\mathrm{F}=6,2^{*} ; \mathrm{C} . \mathrm{V} .=23 \%\right)$, parte aérea $\left(\mathrm{F}=5^{*} ; \mathrm{C} . \mathrm{V} .=7 \%\right)$ e planta toda $\left(\mathrm{F}=18^{*} ; \mathrm{C} . \mathrm{V} .=8 \%\right)$ (Trivelin et al., 2002). Assim como observado por TRIVELIN et al. (2002) para os dados de produção de matéria seca, os tipos de resíduos culturais incorporados ao solo não proporcionaram efeito significativo no acúmulo de macronutrientes, tanto na parte aérea (Tabela 1) como na parte subterrânea (Tabela 2) da cultura durante a safra de cana-planta (11 meses).

O significativo aumento na produção de matéria seca da parte subterrânea (rizomas e raízes) com os níveis de $\mathrm{N}$ resultou, provavelmente, em maior superfície específica de raízes, refletindo em maior exploração do volume de solo e, conseqüente, maior absorção de água e nutrientes. Entretanto, dos nutrientes avaliados na parte aérea da cultura (Tabela 1) apenas o nitrogênio (N) e o enxofre (S) foram acumulados, significativamente, em maiores quantidades em função do aumento das doses de $\mathrm{N}$ aplicadas, tendo este acúmulo comportamento linear (Tabela 1). Para os demais macronutrientes avaliados (potássio - K, fósforo - $\mathrm{P}$, cálcio - Ca e magnésio - Mg) não se obteve resposta significativa de acúmulo na parte aérea da cultura em função das doses de $\mathrm{N}$ aplicadas (Tabela 1). O acúmulo de macronutrientes na parte aérea da cultura seguiu a seguinte ordem decrescente de grandeza: $\mathrm{K}>\mathrm{N}>\mathrm{Ca}>\mathrm{S}>\mathrm{Mg}>\mathrm{P}$ (Tabela 1).

$\mathrm{O}$ efeito sinérgico entre $\mathrm{N}$ e $\mathrm{S}$ em plantas de cana-de-açúcar (variedade NA56 79) foi observado por BATISTA (1977), com interação linear significativa, pois à medida que se aumentou a adubação nitrogenada ocorreu maior acúmulo de $S$ nas plantas. 
Tabela 1. Acúmulo de macronutrientes pela parte aérea da cana-de-açúcar em função da adubação nitrogenada e dos resíduos culturais incorporados no plantio da cultura

\begin{tabular}{|c|c|c|c|c|c|c|}
\hline \multirow[t]{2}{*}{ Tratamentos } & \multicolumn{6}{|c|}{ Parte aérea } \\
\hline & $\mathrm{N}$ & $\mathrm{P}$ & K & $\mathrm{Ca}$ & $\mathrm{Mg}$ & $S$ \\
\hline \multicolumn{7}{|l|}{ Resíduos Culturais $\left({ }^{1}\right)$} \\
\hline $\mathrm{CF}$ & 5.656 & 1.274 & 7.424 & 4.560 & 2.398 & 2.842 \\
\hline SF & 5.490 & 1.259 & 7.303 & 4.237 & 2.117 & 2.833 \\
\hline F resíduos culturais & $0,57^{\mathrm{NS}}$ & $0,08^{\mathrm{NS}}$ & $0,06^{\mathrm{NS}}$ & $0,51^{\mathrm{NS}}$ & $1,64^{\mathrm{NS}}$ & $0,01^{\mathrm{NS}}$ \\
\hline \multicolumn{7}{|l|}{ Doses de $\mathrm{N}\left(\mathrm{mg} \mathrm{vaso}^{-1}\right)$} \\
\hline 0 & 4.868 & 1.218 & 7.032 & 4.027 & 1.758 & 2.398 \\
\hline 900 & 5.093 & 1.290 & 7.294 & 4.270 & 2.224 & 2.645 \\
\hline 1800 & 5.683 & 1.145 & 7.152 & 4.747 & 2.521 & 2.971 \\
\hline 2700 & 6.649 & 1.216 & 7.976 & 4.551 & 2.526 & 3.334 \\
\hline F doses de $\mathrm{N}$ & $12,99^{*}$ & $2,87^{\mathrm{NS}}$ & $0,77^{\mathrm{NS}}$ & $0,49^{\mathrm{NS}}$ & $2,71^{\mathrm{NS}}$ & $11,61^{*}$ \\
\hline F interação de fatores & $1,56^{\mathrm{NS}}$ & $1,54^{\mathrm{NS}}$ & $1,57^{\mathrm{NS}}$ & $0,13^{\mathrm{NS}}$ & $2,15^{\mathrm{NS}}$ & $0,80^{\mathrm{NS}}$ \\
\hline $\mathrm{R}^{2}-$ R.L. & $0,93^{*}$ & NS & NS & NS & NS & $0,99^{*}$ \\
\hline $\mathrm{R}^{2}-\mathrm{R} . \mathrm{Q}$. & NS & NS & NS & NS & NS & NS \\
\hline C.V. $(\%)$ & 10 & 10 & 16 & 25 & 24 & 10 \\
\hline
\end{tabular}

( ${ }^{1}$ CF e SF significam os resíduos culturais (raízes, rizomas, colmos e ponteiros), respectivamente, com e sem folhas secas. NS: não significativo. * significativo a $5 \%$ de probabilidade. $\mathrm{R}^{2}$ : coeficiente de determinação. R.L. e R.Q.: regressão linear e quadrática.

Tabela 2. Acúmulo de macronutrientes pela parte subterrânea da cana-de-açúcar em função da adubação nitrogenada e dos resíduos culturais incorporados no plantio da cultura

\begin{tabular}{|c|c|c|c|c|c|c|}
\hline \multirow[t]{2}{*}{ Tratamentos } & \multicolumn{6}{|c|}{ Parte subterrânea } \\
\hline & $\mathrm{N}$ & $\mathrm{P}$ & K & $\mathrm{Ca}$ & $\mathrm{Mg}$ & $S$ \\
\hline \multicolumn{7}{|l|}{ Resíduos Culturais $\left({ }^{1}\right)$} \\
\hline $\mathrm{CF}$ & 2141 & 274 & 2270 & 1322 & 384 & 376 \\
\hline SF & 2137 & 273 & 2174 & 1262 & 371 & 375 \\
\hline F resíduos culturais & $0,00^{\mathrm{NS}}$ & $0,00^{\mathrm{NS}}$ & $0,16^{\mathrm{NS}}$ & $0,18^{\mathrm{NS}}$ & $0,10^{\mathrm{NS}}$ & $0,00^{\mathrm{NS}}$ \\
\hline \multicolumn{7}{|l|}{ Doses de $N\left(\mathrm{mg} \mathrm{vaso}^{-1}\right)$} \\
\hline 0 & 1516 & 193 & 1627 & 953 & 268 & 261 \\
\hline 900 & 1949 & 252 & 2151 & 1138 & 381 & 347 \\
\hline 1800 & 2513 & 319 & 2786 & 1318 & 390 & 423 \\
\hline 2700 & 2579 & 331 & 2324 & 1758 & 470 & 470 \\
\hline F doses de $\mathrm{N}$ & $7,49^{*}$ & $6,25^{*}$ & $4,04^{*}$ & $6,01^{*}$ & $4,18^{*}$ & $6,73^{*}$ \\
\hline $\mathrm{F}$ interação de fatores & $0,14^{\mathrm{NS}}$ & $0,07^{\mathrm{NS}}$ & $0,53^{\mathrm{NS}}$ & $0,29^{\mathrm{NS}}$ & $0,59^{\mathrm{NS}}$ & $0,51^{\mathrm{NS}}$ \\
\hline $\mathrm{R}^{2}-\mathrm{R} . \mathrm{L}$ & $0,93^{*}$ & $0,94^{*}$ & $0,54^{*}$ & $0,94^{*}$ & $0,91^{*}$ & $0,98^{*}$ \\
\hline$R^{2}-$ R.Q. & NS & NS & $0,89^{*}$ & NS & NS & NS \\
\hline C.V. $(\%)$ & 21 & 23 & 26 & 27 & 26 & 23 \\
\hline
\end{tabular}

( ${ }^{1}$ CF e SF significam os resíduos culturais (raízes, rizomas, colmos e ponteiros), respectivamente, com e sem folhas secas. N. não significativo. * significativo a $5 \%$ de probabilidade. $R^{2}$ : coeficiente de determinação. R.L. e R.Q.: regressão linear e quadrática.

As relações N/S na parte aérea da cultura, cuja amplitude foi de 1,9-2,0, não foram influenciadas pelas doses de N. Em vista disso, pode-se inferir que tendo o solo quantidades adequadas de $\mathrm{S}$, à medida que se aumentou o fornecimento de $\mathrm{N}$ à cultura, as plantas absorveram quantidades proporcionais de $\mathrm{S}$, mantendo, assim, a relação N/S. No entanto, na parte subterrânea esta relação já foi da ordem de 6,0 , três vezes maior que a observada na parte aérea (Tabela 2).
A adubação nitrogenada promoveu aumentos significativos no acúmulo dos nutrientes $\mathrm{N}, \mathrm{P}, \mathrm{K}, \mathrm{Ca}$, $\mathrm{Mg}$ e S na parte subterrânea da cultura, independentemente do tipo de resíduo cultural incorporado ao solo (Tabela 2).

$\mathrm{O}$ acúmulo de macronutrientes na parte subterrânea da cultura seguiu a seguinte ordem decrescente de grandeza: $\mathrm{N}=\mathrm{K}>\mathrm{Ca}>\mathrm{Mg}=\mathrm{S}>\mathrm{P}$ (Tabela 2). 
Para $\mathrm{N}, \mathrm{K}, \mathrm{P}, \mathrm{Mg}$ e $\mathrm{S}$ todos os níveis de fertilização nitrogenada promoveram maiores acúmulos destes macronutrientes na parte subterrânea da planta, em relação à testemunha sem aplicação de $\mathrm{N}$ (Tabela 2). No entanto, para Ca, esse aumento só foi marcante nas doses de 1800 e $2700 \mathrm{mg}$ vaso $^{-1}$ de $\mathrm{N}$ (Tabela 2). Nos acúmulos de nutrientes houve resposta linear à adubação nitrogenada de plantio, com exceção do $\mathrm{K}$, que teve melhor ajuste por uma função quadrática, sendo a dose de $1800 \mathrm{mg}^{\text {vaso }}{ }^{1}$ de $\mathrm{N}$ a que propiciou o maior acúmulo do nutriente (Tabela 2).

Esse mecanismo de maior acúmulo pode ser atribuído a uma translocação dos nutrientes presentes na parte aérea para o sistema radicular da cultura, sendo um reflexo fisiológico da fase de maturação, embora seja consagrado na literatura que os nutrientes cálcio e enxofre são pouco móveis no floema das plantas. Trivelin et al. (1995) levantaram a hipótese da translocação do nitrogênio da parte aérea ao sistema radicular no período entre abril e maio (204 a 237 dias após o inicio do ciclo). Essa translocação gera condições para as plantas emitirem novas raízes, proporcionando assim exploração de maior volume de solo, aumentando o potencial de absorção e acúmulo de nitrogênio do solo. Gava (1999) confirma essa hipótese, tendo em vista que, em seu trabalho, entre abril (169 dias de ciclo) e maio (204 dias de ciclo), na cultura da cana-de-açúcar verificou-se uma queda acentuada no acúmulo de $\mathrm{N}$ na parte aérea das plantas, passando de $120 \mathrm{~kg}$ ha $^{-1}$ de N para $95 \mathrm{~kg} \mathrm{ha}^{-1}$ de N. O acúmulo de $\mathrm{N}$ pela parte aérea só voltou a ser positivo a partir de julho, quando a cultura acumulou $153 \mathrm{~kg} \mathrm{ha}^{-1}$ de N (267 dias de ciclo). Resultados semelhantes foram citados por HAAG et al. (1987) com canaplanta (CB41 76) para o elemento potássio. Observou-se que ocorreu um decréscimo no acúmulo de $\mathrm{K}$ na parte aérea das plantas 14 meses após o plantio, passando de 221 para $194 \mathrm{~kg} \mathrm{ha}^{-1}$ aos 16 meses.

Essas informações da literatura dão subsídios para explicar os maiores acúmulos de $\mathrm{N}$ e $\mathrm{K}$ na parte subterrânea, devido à nutrição nitrogenada. Provavelmente, ocorre ao fim do ciclo da cultura a translocação de $\mathrm{N}$ e $\mathrm{K}$ da parte aérea das plantas para o sistema radicular, objetivando o aumento de suas reservas nutricionais, tendo em vista que a cultura depende dessas reservas para garantir seu próximo ciclo (sucessivas rebrotas).

Recentemente, em experimento desenvolvido em condições de campo, VItTI et al. (2007) constataram o efeito das reservas nutricionais do sistema radicular da cana-de-açúcar (variedade SP 81 3250) na produtividade de colmos do ciclo seguinte. Os autores verificaram aumento linear significativo na produção de colmos em função das quantidades de $\mathrm{N}$ e $\mathrm{S}$ presentes na parte subterrânea (sistema radicular) da cultura no início do ciclo; as quantidades desses nutrientes foram diretamente relacionadas às doses de $\mathrm{N}$ aplicadas no ciclo anterior.

Assim, os resultados deste trabalho evidenciam o efeito sinérgico do nitrogênio no acúmulo dos demais macronutrientes, em especial na parte subterrânea (raízes e rizomas) da cultura. A importância desse acúmulo pode implicar maior longevidade da lavoura canavieira, aliada a ganhos significativos em produtividade de colmos nas rebrotas subseqüentes.

\section{Referências}

AZEREDO, D.F.; BOLSANELLO, J.; WEBWE, H.; VIEIRA, J.R. Nitrogênio em cana-planta - doses e fracionamento. STAB- Açúcar, Álcool e Subprodutos, Piracicaba, v. 4, p. 25-29, 1986.

BASANTA, M.V.; DOURADO NETO, D.; REICHARDT, K; BACCHI, O.O.S.; OLIVEIRA, J.C.M.; TRIVELIN, P.C.O.; TIMM, L.C.; TOMINAGA, T.T.; CORRECHEL, V.; CÁSSARO, F.A.M.; PIRES, L.F.; MACEDO, J.R. Eficiência no uso de nitrogênio em relação aos manejos dos resíduos da cultura de cana-de-açúcar. In: CONGRESSO NACIONAL DA STAB, 8., 2002, Recife-PE. Anais... Recife: Stab, 2002. p. 268-275.

BATISTA, L. F. Aplicação de N, P e ${ }^{35} \mathrm{~S}$ em dois solos de São Paulo e seus efeitos sobre a cana-de-açúcar. 1977. 90f. Dissertação (Mestrado) - Escola Superior de Agricultura “Luiz de Queiroz", Piracicaba, 1977.

CARNAÚBA, B.A.A. O nitrogênio e a cana-de-açúcar. STAB-Açúcar, Álcool e Subprodutos, Piracicaba, v. 8, n. 3, p. 24-41, 1990.

FELLER, U.; FISCHER, A. Nitrogen-metabolism in senescing leaves. Critical Reviews in Plant Sciences, Boca Raton, v.13, n.3, p. 241-273, 1994.

GAVA, G. J. de C. Utilização do nitrogênio da uréia $\left({ }^{15} \mathrm{~N}\right)$ e da palhada $\left({ }^{15} \mathrm{~N}\right)$ por soqueira de cana-de-açúcar no manejo sem despalha a fogo. 1999. 81f. Dissertação (Mestrado) - Centro de Energia Nuclear na Agricultura, Universidade de São Paulo, Piracicaba, 1999.

HAAG, H.P.; DECHEN, A.R.; CARMELLO, Q.A.C. Nutrição mineral da cana-de-açúcar. In: PARANHOS, S. B. (Ed.). Canade-açúcar: Cultivo e utilização. Campinas: Fundação Cargill, 1987. 431p.

KORNDÖRFER, G.H.; VALLE, M.R.; MARTINS, M.; TRIVELIN, P.C.O. Aproveitamento do nitrogênio da uréia pela cana-planta. Revista Brasileira Ciência do Solo, Viçosa, v. 21. p. 23-26, 1997. 
MALAVOLTA, E.; VITTI, G.C.; OLIVEIRA, S.A. Avaliação do estado nutricional das plantas. Piracicaba: Associação Brasileira para a Pesquisa da Potassa e do Fosfato, 1989. 201p.

OLIVEIRA, M.W.; TRIVELIN, P.C.O.; KINGSTON, G.; BARBOSA, M.H.P.; VITTI, A.C. Decomposition and release of nutrients from sugarcane trash in two agricultural environments in Brasil. Proceedings of the Australian Society of Sugarcane Technologist, Brisbane, p. 40, 2002.

ORLANDO FILHO, J.; RODELLA, A.A.; BELTRAME, J.A.; LAVORENTI, N.A. Doses, fontes e formas de aplicação de nitrogênio em cana-de-açúcar. STAB- Açúcar, Álcool e Subprodutos, Piracicaba, v. 17, p. 39-41, 1999.

SILVEIRA, J.A.G.; CROCOMO, O.J. Assimilação de nitrogênio em cana-de-açúcar cultivada em presença de elevado nível de $\mathrm{N}$ e de vinhaça no solo. Revista Brasileira de Fisiologia Vegetal, Campinas, v. 2, p. 7-15, 1990.

VITTI, A.C.; TRIVELIN, P.C.O; GAVA, G.J. de C.; PENATTI, C.; BOLOGNA, I. R.; FARONI, C.E.; FRANCO, H.C.J. Produtividade da cana-de-açúcar relacionada ao nitrogênio residual da adubação e do sistema radicular. Pesquisa Agropecuária Brasileira, Brasília, v. 42, n. 2, 2007. (no prelo)

TRIVELIN, P.C.O.; COLETI, J.T.; LARA CABEZAS, W.A.R. Efeito residual na soqueira de cana-de-açúcar do nitrogênio da uréia aplicada por via foliar na cana-planta. In: SEMINÁRIO SOBRE TÉCNICAS NUCLEARES NA PRODUÇÃO DE PLANTAS AGRÍCOLAS, 1984, Piracicaba. Anais... Piracicaba: CENA, 1984. p.119-124.
TRIVELIN, P.C.O.; VICTORIA, R.L.; RODRIGUES, J.C.S. Aproveitamento por soqueira de cana-de-açúcar de final de safra do nitrogênio da aquamônia $-{ }^{15} \mathrm{~N}$ e uréia $-{ }^{15} \mathrm{~N}$ aplicado ao solo em complemento à vinhaça. Pesquisa Agropecuária Brasileira, Brasília, v. 30, p. 1375-1385, 1995.

TRIVELIN, P.C.O.; VICTORIA, R.L.; RODRIGUES, J.C.S. Utilização por soqueira de cana-de-açúcar de início de safra do nitrogênio da aquamônia $-{ }^{15} \mathrm{~N}$ e uréia $-{ }^{15} \mathrm{~N}$ aplicado ao solo em complemento à vinhaça. Pesquisa Agropecuária Brasileira, Brasília, v. 31, n. 2, p. 89-99, 1996.

TRIVELIN, P.C.O.; VITTI, A.C.; OLIVEIRA, M.W.; GAVA, G.J.C.; SARRIÉS G.A. Utilização de nitrogênio e produtividade da cana-de-açúcar (cana-planta) em solo arenoso com incorporação de resíduos da cultura. Revista Brasileira de Ciência do Solo, Viçosa, v.26, n.3, p.636-646, 2002.

URQUIAGA, S.; CRUZ, K.H.S.; BODDEY, R.M. Contribution of nitrogen fixation to sugar cane: nitrogen-15 and nitrogenbalance estimates. Soil Science Society of American Journal, Madison, v. 56, p. 105-114, 1992. 